\title{
Evolution naturelle d'un remplissage sédimentaire intramontagnard et impacts des aménagements contemporains : L'exemple de la vallée de l'Arve (74)
}

\author{
J.-L. Peiry J.-P. Bravard \\ Département de Géographie, Université Lyon 3 \\ 74, rue Pasteur, 69365 Lyon
}

\begin{abstract}
La gestion des sédiments fluviatiles est devenue depuis quelques années l'une des préoccupations des ingénieurs hydrauliciens et gestionnaires de cours d'eau (Séminaire national de Propriano, 1981). Les problèmes que pose la sédimentation dans les retenues hydro-électriques, les graves désordres observés sur la plupart des cours d'eau des Alpes et de la périphérie alpine mobilisent les chercheurs et nécessitent souvent d'importants et coûteux travaux (Vivian et Thomas, 1982). L'objectif de cet article est de montrer que les dépôts remblayant les vallées alluviales constituent une ressource rapidement épuisable et non renouvelable à court et même moyen terme.
\end{abstract}

Ils sont le reflet de l'histoire complexe du remplissage sédimentaire de ces vallées au cours des derniers millénaires. L'étude de cet héritage sédimentaire s'avère indispensable, car très souvent il influe sur les processus fluviaux contemporains et perturbe la dynamique des sédiments.

L'Arve en fournit un exemple démonstratif; ce cours d'eau des Alpes du Nord à charge graveleuse prend sa source dans les hautes montagnes englacées du MontBlanc et conflue avec le Rhône peu en aval du lac Léman (fig. 1). La vigueur du relief, l'abondance des précipitations conditionnent des écoulements fortement énergiques, au régime contrasté. Ce cadre géographique montagnard permet d'expliquer la forte activité de la dynamique fluviale : elle se traduit par la réponse brutale et marquée du cours d'eau à toute perturbation, même mineure, des flux hydriques et sédimentaires.
1. Les plaines alluviales intramontagnardes: un potentiel à géométrie variable

Le phénomène d'incision accélérée du lit des rivières est enregistré dans de nombreuses vallées alpines (PEIRY, 1987, 1987 a). Ce phénomène n'est pas nouveau à l'échelle des temps géologiques et historiques ; il prend simplement une ampleur exceptionnelle face à l'intensité de la perturbation engendrée par l'homme.

Les plaines alluviales intra-alpines constituent la partie visible d'un remplissage sédimentaire souvent épais de plusieurs dizaines de mètres, voire davantage, établi dans des cuvettes de surcreusement glaciaire. La variété des faciès sédimentaires : moraines, argiles lacustres, cailloutis fluvio-glaciaires ou fluviatiles, etc... traduit la succession de plusieurs types de remplissage imbriqués de manière complexe. Le plancher alluvial moderne est l'état le plus récent d'une série de phases holocènes caractérisées par un intense remaniement fluviatile des dépôts de remplissage.

Au cours de l'holocène, certaines vallées ou tronçons de vallée ont connu un type d'évolution discontinu, marqué par des phases successives d'exhaussement du plancher alluvial ou, au contraire, de déstockage sédimentaire par incision fluviatile sans négliger le rôle des conditions géologiques régionales, telles que les phénomènes de subsidence. L'étude des cours d'eau d'Europe Centrale a permis de démontrer que ces variations verticales enregistrent les fluctuations de la dynamique fluviale par l'intermédiaire de

Natural evolution of an intramontane alluvial infill and impacts of contemporary development. The example of the Arve valley.

The present valley floors of rivers in mountainous regions represent a particular phase of postglacial and holocene dynamics. Over an extended period i.e. at a geological or historical timescale, alluvial masses have experienced changes in their facies and volume, which are linked to change in the character of mountain erosion and fluvial dynamics. At a contemporaneous timescale, floodplains have probably experienced a shortage of sediment inputs : the resulting degradation of the river bed was canalized by nineteenth century enbankments and gravel extraction. These rapid changes have affected the long profiles and reduced the transport of bedload; conversely, the movement of suspended matter appears to have been less affected. 


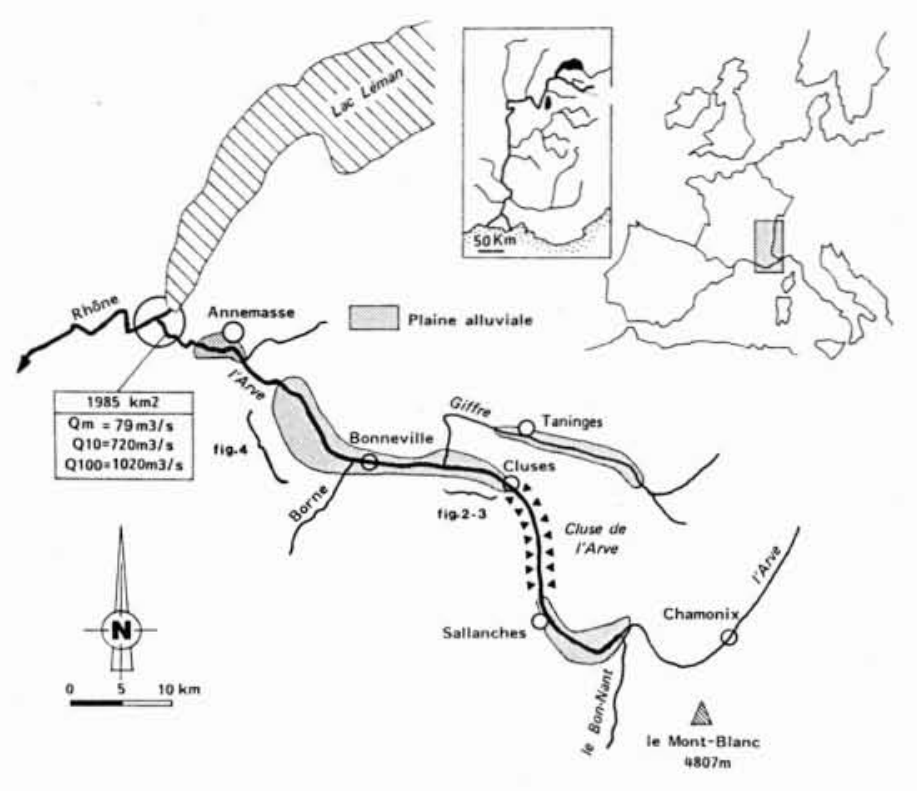

1. Localisation de l'espace d'étude.

la réponse du cours d'eau aux changements de la charge et du débit dans le bassin versant (STARKel, 1983). L'origine des variations de flux fut d'abord naturelle avant le Néolithique, principalement sous l'effet des changements climatiques modifiant les bilans hydriques et l'activité morphogénique sur les versants. Depuis cette époque, leur origine est aussi anthropique, l'homme perturbant d'abord de manière indirecte les flux d'eau et de sédiments (défrichements des versants), puis plus récemment exerçant un contrôle direct de l'activité fluviale (endiguements, régulation du régime, extractions...).

Ajoutons que certaines variations verticales mineures peuvent être la manifestation de déséquilibres temporaires internes aux lits fluviaux.

Au cours des derniers millénaires, les plaines de l'Arve ont connu des phases de fluctuation verticale du plancher alluvial complexes, d'une amplitude d'environ 15 mètres. Elles ont été accompagnées de variations des faciès sédimentaires caractérisant le type de cours d'eau et la nature de sa dynamique. Ainsi, sur l'Arve moyenne, des dépôts fins étendus et épais de plusieurs mètres mis en place avant 8600 ans BP par une rivière peu énergique ont été fossilisés par environ 5 mètres d'alluvions grossières qui témoignent d'une forte activité fluviale (PEIRY, 1988, p. 60 et s.). A l'échelle historique, le plancher alluvial a aussi connu des fluctuations verticales dont deux ont été repérées :

1) Au confluent Giffre-Arve (fig. 1), la découverte de piquets de bois datés de la fin du XIII ${ }^{\mathrm{e}}$ siècle, plantés dans les sédiments fins holocènes indique une tendance au déstockage sédimentaire des cours d'eau dans le bassin de Cluses (PEIRY, 1988, p. 262 et s.). D'après LAMB (1984), la période 1150-1300 ans après J.-C. apparaît être celle de l'optimum climatique du Moyen-Age en Europe Occidentale. Le climat régulier, chaud et relativement sec de cette époque aurait influé sur la balance hydrique, probablement négative, et la fourniture sédimentaire des versants, moins abondante qu'aujourd'hui en raison d'une meilleure végétalisation des pentes.

2) $\mathrm{Au} \mathrm{XVII}^{\circ}$ siècle, de fréquentes crues des émissaires glaciaires ont favorisé l'ennoiement du fond de vallée de Chamonix sous les sédiments grossiers. Au XVIII siècle, la bande de tressage s'élargit rapidement dans le bassin de Cluses car la plaine s'exhausse progressivement sous l'effet des apports amont. Plusieurs centaines d'hectares de terre agricole sont rendus à la rivière ou transformés en marécages (PeIRY, 1987 b). Cette phase de recrudescence de l'activité fluviale est sans doute liée à la péjoration climatique du Petit Age Glaciaire et, dans une proportion inconnue, à l'influence des activités humaines sur le bassinversant. Ainsi, les plaines alluviales de l'Arve enregistrentelles la lente descente vers l'aval de millions de $\mathrm{m}^{3}$ de gravier et de sable; l'Arve établit une nouvelle géométrie de son lit, un nouveau profil en long adaptés au transport de cette charge accrue. Les plaines alluviales sont donc des espaces de stockage qui diffèrent et atténuent la réponse des cours d'eau vers l'aval. Ce stockage est assuré par la mise en place de "mégaformes » de sédimentation ; elles contrôlent le plancher alluvial du $\mathrm{xIx}^{\mathrm{e}}-\mathrm{xx}^{\mathrm{e}}$ siècle, celui sur lequel s'exerce la dynamique fluviale contemporaine.

\section{Les effets de l'homme sur la dynamique des sédi- ments}

\subsection{Les endiguements longitudinaux insubmersibles}

Dès le $\mathrm{XIX}^{\mathrm{e}}$ siècle, l'homme devient un facteur fondamental de contrôle de l'activité fluviale par ses interventions directes sur le cours d'eau. Les endiguements longitudinaux insubmersibles, établis dans les années 1820-1840 par sections le long de la rivière, ont favorisé d'importants remaniements sédimentaires; ils sont à l'origine d'une nouvelle géographie des dépôts de la plaine alluviale.

Dans les secteurs endigués, la concentration des écoulements, la modification des pentes du lit résultant du recoupement des sinuosités naturelles ont provoqué un rééquilibrage du lit. Il s'est manifesté par des processus de basculement du profil en long, donnant lieu à une recrudescence de l'activité des charriages, phénomène classique sur les rivières à fond graveleux (GEMAEHLING et ChABERT, 1962 ; Winghart et ChABERT, 1965 ; BraVARD, 1987). Ainsi, dans la partie amont de la section endiguée de Bonneville, la pente de la ligne d'eau mesurée à 0,0027 après les travaux de correction du début des années 1840 diminua progressivement à 0,0019 en 1854 . Cet ajustement provoqua une incision de $2 \mathrm{~m} 40$ à l'extrémité amont des digues (MORTILLET, 1856). Les matériaux arrachés au lit amont vinrent s'accumuler dans la partie inférieure de la section endiguée. L'exhaussement du lit au voisinage de Bonneville se manifesta par une submersion de plus en plus fréquente des digues, la pente se réduisant en quelques années à 0,0013 (1854). Au débouché des digues, les trans- 
ports solides accrus par la déstabilisation du chenal amont. le changement brutal des sections d'écoulement, favorisèrent l'édification d'un cône de déjection : la rivière à l'instabilité latérale croissante élargit vigoureusement son lit, en se jetant contre les propriétés riveraines qu'elle érodait : chaque nouvelle crue favorisait l'ouverture de nouveaux bras et l'abandon des anciens chenaux par "avulsion ".

L'impact des travaux d'endiguement sur la dynamique de la charge fine transitant en suspension est plus difficile à cerner. Au moment de l'édification des digues longitudinales, la rivière qui tressait vigoureusement occupait un lit très large et les environnements calmes de plaine d'inondation susceptibles d'accueillir les dépôts fins ètaient peu nombreux. Lors de l'endiguement, le gain de terrain s'est produit essentiellement au détriment de la bande d'activité fluviale graveleuse, incapable de piéger durablement la charge fine. Contrairement au Haut-Rhône, oủ l'endiguement en réduisant les divagations du fleuve a favorisé la sédimentation fine de plaine d'inondation (BRAVARD, 1987), l'endiguement insubmersible a réduit la capacité de stockage de la plaine alluviale. Cependant, cette réduction de la capacité de stockage, déjà faible à l'état naturel, est demeurée modeste.

\subsection{Les extractions de gravier}

Depuis quelques décennies, de très nombreuses perturbations d'origine anthropique affectent la dynamique des flux d'eau et surtout des sédiments. Excepté sur de courtes sections de rivière, les débits ont été peu modifiés par les aménagements hydroélectriques de l'Arve demeure l'un des cours d'eau des Alpes du Nord les moins perturbés (Edouard et Vivian, 1984). Le changement de la dynamique des sédiments est par contre profond et affecte aussi bien les matériaux charriés que les matériaux transitant en suspension. Ce changement a parfois une origine indirecte. comme par exemple la modification de la fourniture sédimentaire alimentant l'Arve, issue des affluents montagnards et des torrents. Cependant, la responsabilité du changement incombe avant tout aux extractions de gravier qui ont rapidement excédé la charge annuelle estimée à $150000 \mathrm{~m}^{3} / \mathrm{an}$. Elles se développèrent au cours de la première moitié du $\mathrm{xx}^{\mathrm{e}}$ siècle sur l'Arve suisse, dans l'agglomération de Genève; dans la plaine alluviale amont, leur ouverture, plus tardive, date des années 1950. Après une période d'intense activité pendant la décennie 19601970 , le nombre d'exploitations a progressivement diminué ; les autorisations d'extraire dans le lit ne sont plus renouvelées depuis 1983. Au total, c'est plus de $15 \mathrm{M}$ de $\mathrm{m}^{3}$ de matériaux alluvionnaires qui ont été extraits du lit de l'Arve, dans toutes les sections de sa plaine alluviale.

Le principal impact des extractions est la profonde perturbation de la dynamique des charriages qui se sont progressivement raréfiés. Elle s'est traduite par un ajustement des formes du chenal à cette pénurie de charge grossière : le chenal large à bras multiples a disparu, au profit d'un lit unique, étroit et rectiligne, profondément incisé dans la plaine alluviale de tressage. L'incision du chenal, quasiment continue sur l'ensemble du cours de l'Arve a, en certains points, atteint des valeurs record (PeIRY, 1987 a) : en tête du bassin de Sallanches, la chute de la ligne d'eau depuis 1950 est voisine de $12 \mathrm{~m}$; dans la moyenne vallée de l'Arve, près de Cluses, l'incision dépassait une profondeur de $8 \mathrm{~m}$ avant l'aménagement du lit intervenu en 1983 (fig. 2). Sur ce tronçon de rivière, la pente moyenne de la ligne d'eau a notablement diminué, passant de 0,0028 avant impact à 0,0018 en 1981. La tendance à la décroissance de la pente du chenal, observée également sur d'autres sections de la rivière, résulte d'un ajustement des paramètres décrivant la géométrie du lit en réponse aux changements des transports sédimentaires. Le rôle des dragages dans l'évolution du profil en long est double :

1) l'augmentation des pentes locales au droit des zones d'extraction a favorisé la déstabilisation du lit par des processus d'érosion régressive ;

2) en piégeant les graviers charriés par l'Arve, les souilles d'extraction ont provoqué un déficit d'apports aux sections aval. Ce déficit a été en partie comblé par la reprise en charge des sédiments jusqu'alors stockés dans le lit.

Dans la moyenne vallée de l'Arve, l'ampleur de l'incision résulte également de la présence, à faible profondeur sous les graviers holocènes, d'une puissante formation fine (fig. 2).

Ces dépôts fins, résistant mal à l'érosion, ont été rapidement disséqués par la rivière ; de plus, leur granulométrie a accentué l'affaiblissement des pentes d'équilibre dans les secteurs où ils affleurent.

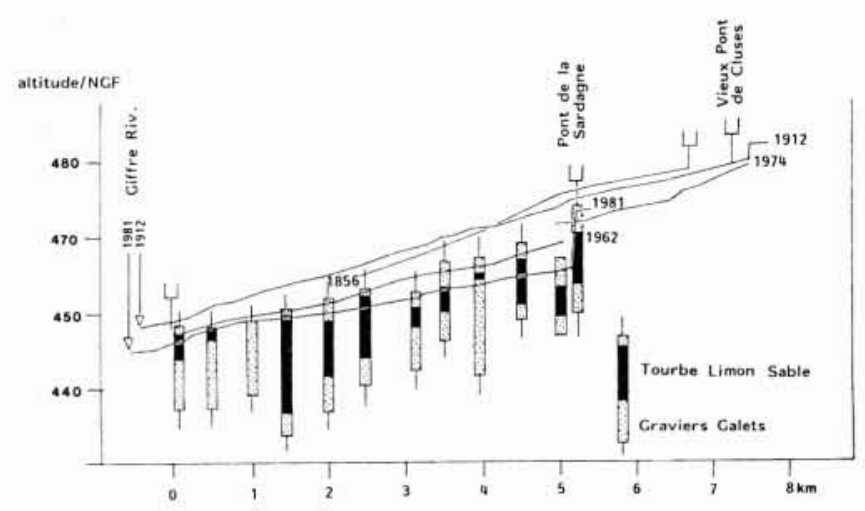

2. Evolution récente du chenal de l'Arve dans le bassin de Cluses. L'incision a dégagé une importante formation fine holocène.

Les extractions de gravier ont également perturbé la dynamique des sédiments fins. Contrairement aux matériaux charriés, ils transitent facilement et parcourent de longues distances sans être piégés dans les souilles d'extraction. 


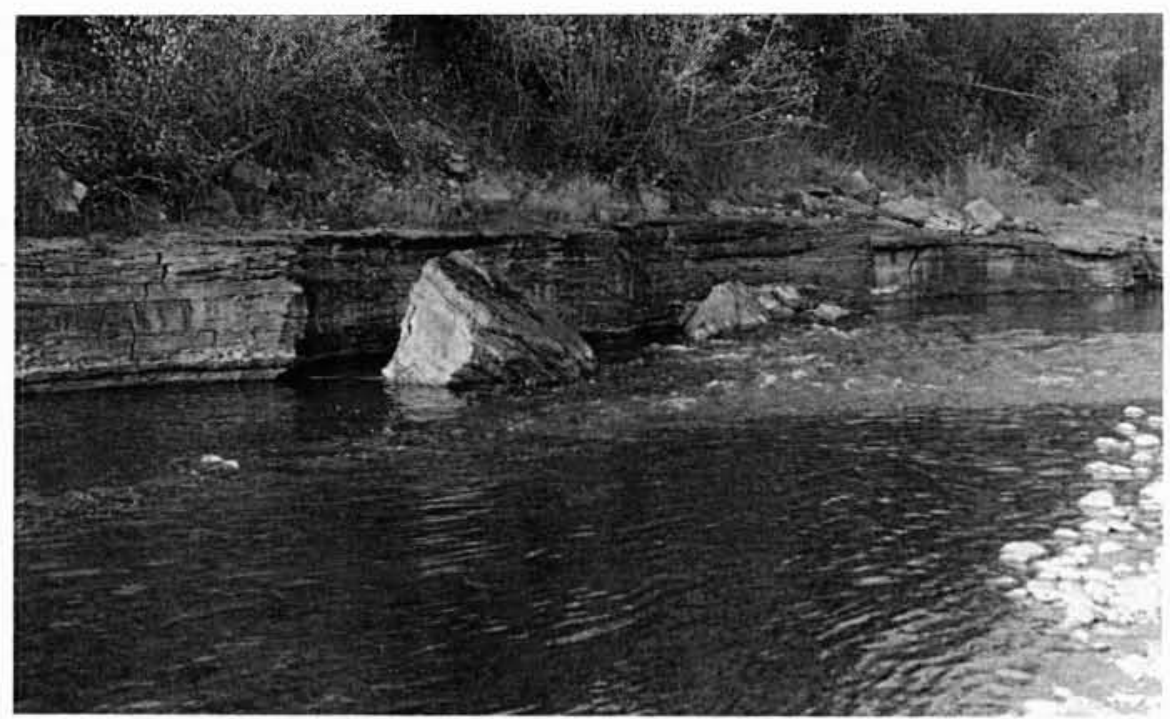

3. Formations fines holocènes incisées par la rivière : sapées à leur pied, elles basculent par paquets dans le chenal, sont transportées sur de courtes distances sous la forme de galets d'argiles, puis rapidement désagrégées et remises en suspension.

4. La basse-Arve en aval de Bonneville. Au tressage actif de 1730 s'est substitué un lit à chenal unique bordé de gravières en eau. La rupture partielle des digues isolant le plan d'eau a permis la formation d'une sédimentation grossière dans l'axe du chenal et une sédimentation fine de type plaine d'inondation.

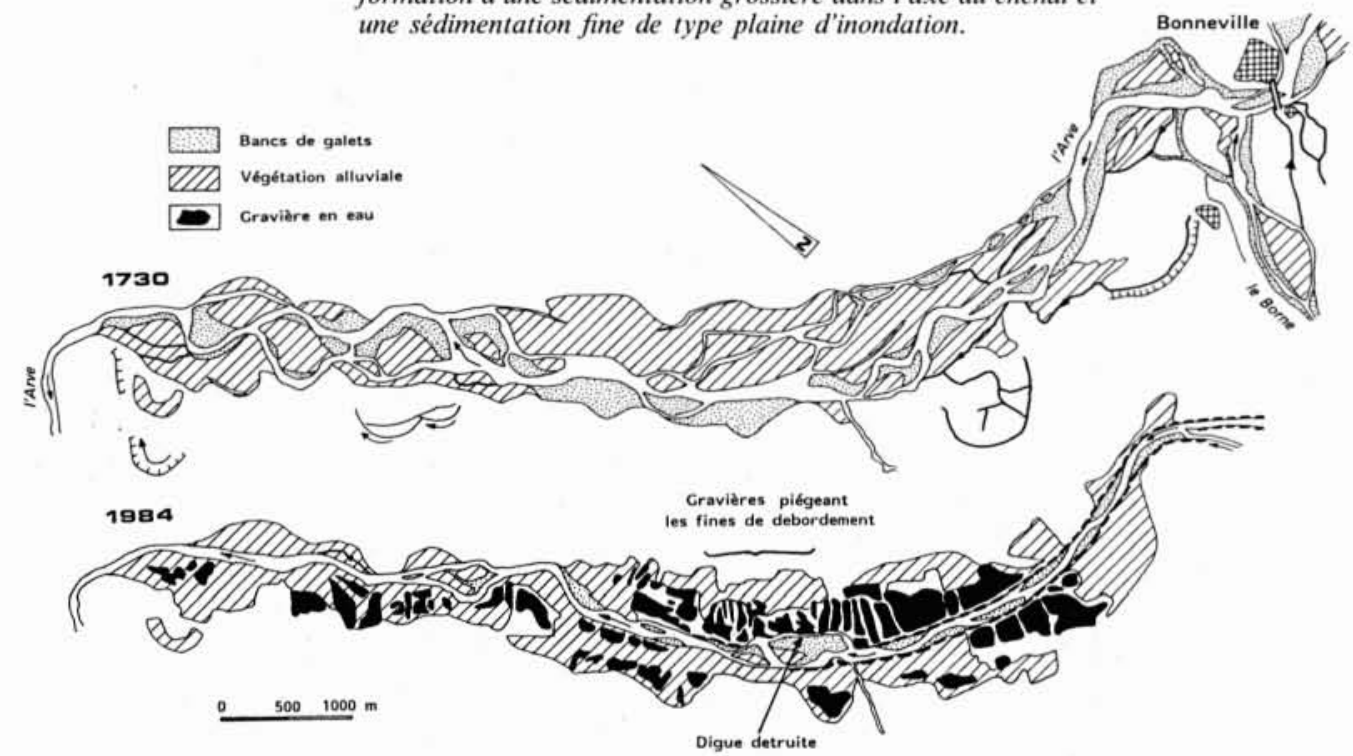

La profonde incision de la rivière a favorisé la reprise en charge d'importantes quantités de fines. L'origine de ces sédiments est double :

1) il s'agit, pour une part difficile à évaluer, de la matrice sableuse des graves constituant le plancher alluvial et ayant échappé aux prélèvements ;

2) ces matériaux sont également issus de l'entaille des formations sablo-limoneuses sous-jacentes qui constituent aujourd'hui le lit de l'Arve dans le bassin de Cluses (fig. 2 et 3).

Ainsi dans cette section du cours d'eau, le déstockage de la plaine alluviale atteint $4 \mathrm{M}$ de $\mathrm{m}^{3}$, alors que le volume total des extractions me semble guère avoir dépassé $2 \mathrm{M}$ de $\mathrm{m}^{3}$. Un phénomène identique a été observé sur le Fier supérieur (SOGREAH, 1982). Une autre conséquence de l'incision est la suppression, sur la presque totalité du cours de l'Arve, des zones soumises à l'inondation. La diminution de la surface et de la fréquence d'inondation des plaines de l'Arve a réduit la capacité du lit majeur à stocker la charge fixe. Il est curieux de constater qu'actuellement les principales aires de stockage de la charge fine résultent d'un effet d'impact : de très nombreuses zones d'extraction ont été ouvertes dans l'ancienne plaine d'inondation de tressage, à l'abri de digues composées de matériaux alluvionnaires (fig. 4). Leur récente rupture a mis les casiers d'extraction directement en contact avec la rivière et a favorisé la sédimentation de matériaux fins en marge du chenal. En dépit de ce cas particulier récent et très local, il apparaît que les matériaux fins remis en suspension transitent intégralement jusqu'au Rhône sans quaaucun stockage de grande ampleur ne soit possible. 


\section{Conclusion}

Par son ampleur et sa rapidité, l'incision de l'Arve au cours des 30 dernières années indique une perturbation extrêmement profonde de la dynamique des transports solides. Ce sont les actions de l'homme sur les flux d'eau et de sédiments qui permettent d'expliquer cette rupture géomorphologique. Plusieurs points sont à retenir :

1) tout d'abord la brutalité et l'ampleur des déséquilibres touchant les cours d'eau alpestres, renforcés par le caractère très énergique de l'environnement montagnard ;

2) ensuite l'origine multiple des déséquilibres: ils peuvent provenir de perturbations directes de l'environnement fluvial, à proximité du lieu où le changement est perçu (endiguement, extraction de gravier dans le lit...); ils peuvent aussi être la conséquence de perturbations plus lointaines, que transmettent à distance les flux d'eau et de sédiments ;

3) l'analyse des sédiments de l'Arve à travers l'évolution de sa géomorphologie sur plusieurs centaines d'années, voire plusieurs millénaires, montre que les changements contemporains accélérés ne sont pas un phénomène sans précédent. Les dépôts sédimentaires des plaines alluviales sont une mémoire de l'histoire complexe du cours d'eau. Les variations dans le temps des flux d'eau et de matières sont à l'origine de phases d'accumulation, auxquelles ont succédé des périodes de déstockage sédimentaire. La dynamique contemporaine des sédiments est influencée non seulement par les processus actuels, mais aussi par des dépôts anciens témoignant d'autres conditions hydrodynamiques et de fourniture sédimentaire.

Il apparaît donc que toute action de gestion des sédiments fluviatiles et plus généralement de l'espace fluvial doit être fondée sur une double analyse de la dynamique des cours d'eau, à la fois dans l'espace (recensement des facteurs influençant la dynamique à l'échelle du bassin versant) et dans le temps (évolution de la dynamique sur plusieurs centaines, voire plusieurs milliers d'années). L'aménageur doit conserver à l'esprit que les cours d'eau ont une grande capacité à transmettre les changements, en particulier vers l'aval, d'où la nécessité de développer une gestion globale de la rivière. Il doit enfin prendre conscience du fait que les ressources en graviers des plaines alluviales ne sont pas indéfiniment renouvelables. Les extractions se font au détriment d'un stock lentement accumulé et non renouvelable à court et même à moyen terme.

\section{Bibliographie}

Bravard, J.-P. (1987), Le Rhône du Léman à Lyon. La Manufacture éd. Lyon, 452 pages.

EDOUART, J.-L., H. Vivian (1984), Une hydrologie naturelle dans les Alpes du Nord? Revue de géographie alpine, p. 72, 257-278.

Gemaehling, C. et J. Chabert (1962), Transports solides et modification du lit d'une rivière en forte pente: la Drôme. AIHS, Bari (1962), p. 59, 259-272.

LAMB, H. H. (1984), Climate in the last thousand years : natural climatic fluctuations and changes. In " The climate of Europe: Past, Present and Future ». H. Floher et R. Fantechi èd., D. Reidel Publishing Company, Dordrecht, p. 25-64.

Mortillet, G. (1856), Diguement des rivières torrentielles des Alpes et plus spécialement de l'Arve. Revue savoisienne, p. 145165.

PEIRY, J.-L. (1987 a), Channel degradation in the middle Arve river (France). Regulated rivers, 1/2, p. 183-188.

PEIRY, J.-L. (1987 b), Dynamique fluviale historique de l'Arve dans le bassin de Cluses (Haute-Savoie). $112^{\mathrm{e}}$ Colloque Natio- nal des Sociétés Savantes, CTHS section de géogr. Lyon, 1987, 15 pages (sous presse).

PEIRY, J.-L. (1988), Approche géographique de la dynamique spatiotemporelle des sédiments d'un cours d'eau intra-montagnard: exemple de la plaine alluviale de l'Arve (Haute-Savoie). Thèse Université Lyon III, 378 pages.

Séminaire national de Propriano (1981), Doc BRGM n 30.

SOGREAH (1982), Etude hydraulique de la protection du CD 909 contre les crues du Fier entre le pont de Morette et Alex. Rapport d'ingénieur non publié $\mathrm{n}^{\circ} \mathrm{R} 36.1579,31$ pages et plans.

STARKEL, L. (1983), The reflexion of hydrologic changes in the fluvial environment of the temperate zone during the last 15000 years. In "Background to palaeohydrology" K. J. Gregory éd., John Wiley et Sons, Clichester, p. 213-235.

Vivian, H., A. Thomas (1981), Erosion et transports solides dans le bassin du haut-Drac. Rapport d'étude, CEMAGREF, Grenoble, 186, 105 p.

Winghart, J. et J. Chabert (1965), Haut-Rhône à l'amont de Lyon: étude hydraulique de l'île de Miribel-Jonage. La Houille Blanche, p. 7, 1-20.

Remerciements : Cette étude a bénéficié de l'appui matériel du "sous-groupe Arve " mis en place par la Société hydrotechnique de France. Que M. Merle, directeur du SRAE Rhône-Alpes et Mme Sanchis en soient remerciés, ainsi que MM. Cogoluenhes (chef du service de RTM de Haute-Savoie), Blanc (directeur départemental de l'Equipement de Haute-Savoie) et leurs collaborateurs. 